\title{
Research on Maritime Emergency Material Dispatch Based on Beetle Antennae Search Algorithm
}

\author{
*JIANG Hongzheng ${ }^{1, \mathrm{a}}$, ZHU Yuzhu ${ }^{2}$ \\ ${ }^{1}$ Navigation College of Dalian Maritime University master student Dalin, China \\ ${ }^{2}$ Navigation College of Dalian 2Maritime University full professor Dalin, China
}

\begin{abstract}
The research on dispatching of emergency supplies for maritime emergencies is the key to improve the use efficiency of marine emergency resources and the success rate of search and rescue. In view of the characteristics of emergency accidents at sea, in this paper, a material scheduling model with the shortest time is built for multi-accident points, multi-ship dispatching and multi-material supply points, and the optimal material scheduling scheme is obtained. The step-size updating coefficient eta is introduced into the variable step-size optimization search algorithm ( BAS ), so that the step-size decreases with the depth of optimization, which improves the searching precision and accuracy of the search algorithm. The simulation experiment verifies that this algorithm can solve the optimization scheme of material scheduling quickly and efficiently.
\end{abstract}

\section{Introduction}

In recent years, associated scholars have conducted a lot of research on the dispatching problem of maritime emergency supplies and related intelligent optimization algorithms. Taking into account the satisfaction of the demand point on the acceptance of materials and the transportation cost, Fuyu Wang and Jie Ding conducted simulation of an emergency in a certain area in 2019 [1], first applied the BAS algorithm to the study of emergency material scheduling, and compared the BAS algorithm with the POS algorithm to verify the rationality of the algorithm in solving the problem of emergency material dispatch. In 2011, Jing Wang[2] used genetic algorithm to study the problem of multi-material dispatching in Marine oil spill accidents, and introduced the concept of dynamic accident point against the characteristics of marine oil spill accidents, which laid a foundation for future research on marine emergency material dispatching. Bo Shen[3] established a LABAS algorithm model based on Lévy flight and adaptive strategy, improving the convergence speed, stability and local development capacity of the algorithm. In 2011, Jun Wang[4] carried out correlational research on the responsibility of water rescue resource allocation and the stability of synergetic mechanism, which laid a foundation for reasonably determining the attribution of accident liability, determining the allocation level of regional rescue resources and its apportionment among the responsible subjects. Tingting Chen and He Yin et al[5] put forward a particle swarm optimization algorithm based on the beetle antennae search, which combined the advantages of the BAS and the POS algorithms to improve the search speed and precision of the algorithm, and they applied it to the portfolio model containing the complete cost. Based on the analysis of the influencing factors of emergency resource scheduling, Meirong Wang [6] determined the scope of the target, and on this basis, established a combination optimization model of aggregation point selection based on reliability and a path selection model based on temporal planning. Hui Zhao and Mengya Hao et al.[7] proposed a global path planning algorithm ( BACA *) based on the combination of beetle antenna search algorithm and $\mathrm{A} *$ algorithm, which solved the problems of poor local path, slow convergence, and more breakpoints in path planning. Xuebin Zhu and Jing Lv[8] proposed a maritime emergency material dispatching model with a single accident point and multiple emergency bases, providig theoretical support for the formulation of strategies for maritime emergency material dispatching. Considering the economic cost and penalty cost, Qingchen Sun and Linchi $\mathrm{Qu}[9]$ established a stochastic programming model to optimize the location of the emergency material storage warehouse and the inventory of different kinds of materials, proposed the sample mean approximation method, and conducted an example simulation to verify the rationality of the algorithm.

In conclusion, the BAS algorithm has not been applied to the research on maritime emergency material dispatching in the current correlational research. Therefore, in this paper, the shortest time optimization model of maritime emergency supplies scheduling is established, and the variable step size BAS algorithm is used to solve the model. In addition,

\footnotetext{
a*614316245@qq.com
} 
compared with the traditional BAS algorithm, the superiority of the algorithm is verified, so as to obtain the optimal materials dispatching scheme, which improves the emergency response speed and provides a method for quickly providing material materials dispatching scheme.

\section{Problem description and modeling}

After an accident occurs at sea, the port usually arranges the ship to establish the corresponding "base station" at the accident point and the predicted target drift position. Therefore, under the condition of multi-accident points, multi-dispatch vessels and multi-supply points, an emergency material dispatching model is established in this paper, and the BAS algorithm is used to solve the optimal scheduling scheme.

Model hypothesis:

(1) The location of each accident point and the material demand interval are known.

(2) The reserves of each supply point are greater than the demand of the accident point.

(3) Since the loading and unloading time of each ship is not much different, the loading and unloading time of the ship is not considered in this paper, and only the shortest transportation time is sought.

(4) In this article, only a single class of goods is considered.

Description of the model:

$G=\left\{g_{i} \mid i=1,2,3, \ldots, m\right\}$ represents the supply point collection, and $Q=\left\{q_{i} \mid i=1,2,3, \ldots, n\right\}$ represents the accident point demand collection. $I_{n}(A)$ is the material demand interval of each accident point, $S=$ $\left\{s_{i} \mid i=1,2,3, \ldots k\right\}$ represents the ship assembly, including the ship carrying capacity $d(t)$, and ship speed $v$ ( $n$ mile $)$,

$X_{m \times n}$ represents the distance element between each supply point and each accident point, $x_{m n}$ 表 represents the distance ( $n$ mile) between the material supply point $m$ and the accident point $n$. The time for Ship $k$ to complete dispatching is $t_{k}$, which is $T=\left\{t_{i} \mid i=1,2,3, \ldots, k\right\}$. The objective function of the algorithm is to calculate the minimum value of the maximum dispatching time of each ship in $T$. The objective function and constraint conditions are as follows:

$$
\begin{gathered}
F=\min \left(\max \left(t_{i}\right)\right) \\
\sum_{i=1}^{m} g_{i}>\sum_{i=1}^{n} q_{i} \\
\sum_{i=1}^{m} g_{i n} \in I_{n}(A) \\
t_{m n k}=x_{m n} / v_{k} \\
C_{k}(q)=G_{k}(q)
\end{gathered}
$$

Formula (1) represents the objective function of finding the shortest scheduling time, Formula (2) indicates that the material quantity of each material supply point is greater than the material demand of each accident point, Formula (3) indicates that the total amount of materials delivered by each supply point to the accident point $n$ meets the material demand range of the accident point $n$, Formula (4) represents the time of ship $k$ from $m$ to $n$, Formula (5) indicates that the quantity of materials dispatched by any ship each time is equal to its carrying capacity.

\section{Introduction and steps of BAS algorithm}

Introduction of BAS algorithm: BAS algorithm is a new optimization search algorithm proposed in 2017, which is similar to genetic algorithm in that neither of them needs to know the specific form and gradient information of the objective function. Compared with particle swarm optimization (PSO), when BAS algorithm is used to search, only one longhorn beetle is needed. When searching for food, longhorn beetles do not know the exact location of the food at first. They search for food by the intensity of the smell they receive from their two tentacles, moving to the stronger side each time. Longhorn beetles can efficiently search for food with this method. The basic process of BAS algorithm is as follows:

(1) The initial position of longicorn beetles is generated randomly.

(2) The direction random vector of the two antennae of longicornis longicornis was established for normalization.

(3) The coordinates of the two antennae of longicorn beetles were calculated.

(4) According to the objective function, the odor intensity of the two tentacles of longhorn beetle was obtained.

(5) According to the odor intensity, the next position of longhorn beetles was determined.

(6) The above steps are repeated for many times to obtain the optimal solution of the objective function.

Algorithm steps:

(1) Initial value generation

According to the number of shipping vessels $n$ and the number of accident points $m$, the initial matrix $B_{m \times n}$ is generated, in which the elements of the matrix represent the quantity of materials transported by each ship and should meet the constraint conditions. If not, the initial matrix is regenerated.

( 2 ) Direction vectors of longicorn tentacles are generated and normalized

$$
\operatorname{dir}=\frac{\operatorname{rands}(m, n)}{\|\operatorname{rands}(m, n)\|}
$$

(3) Calculation of coordinate of left and right antennae of longicorn

$$
\begin{aligned}
& x_{L}=x_{z}+d_{0} \times \operatorname{dir} / 2 \\
& x_{R}=x_{z}-d_{0} \times \operatorname{dir} / 2
\end{aligned}
$$


In Formula (7), $x_{L}$ represents the coordinates of the left antenna, $x_{R}$ represents the coordinates of the right antenna, $d_{0}$ represents the distance of the measuring antenna, and $x_{z}$ represents the centroid coordinates of th $z$-iteration of the longhorn beetle.

(4) Calculation of odor intensity of the left and right antennae of longicorn

Bring the left and right antenna coordinates into the objective function.

$$
\begin{aligned}
& f_{L}=f\left(x_{L}\right) \\
& f_{R}=f\left(x_{R}\right)
\end{aligned}
$$

In Formula $(8), f_{L}$ and $f_{R}$ respectively represent the odor intensity of the left and right antennae of longicorn beetles, namely, the objective function value.

(5) The odor intensity of left and right antennae is compared to determine the next location of longhorn beetle

The objective function of modeling is to find the minimum of the total scheduling time, so the next location of longhorn beetle moves to the direction of weak odor.

$$
\begin{gathered}
x_{n e x t}=x_{z}-\operatorname{step} \times \operatorname{dir} \times \operatorname{sign}\left(f_{L}\right. \\
\left.-f_{R}\right)
\end{gathered}
$$

In Formula (9), $x_{\text {next }}$ represents the next position of longhorns longicorn, step represents the step size of longicorn, and the initial value of the set step size should be as large as possible.

(6) A revision of the step size

In the process of searching the optimal solution of longicorn beetles, the step size of the longicorn beetles should be revised, so that the algorithm has better global search ability in the early stage and certain local search ability in the later stage. Therefore, in the whole search process, the step size of longicornis longicornis should be continuously reduced until the optimal solution is found. In other words, when a more optimal solution is found, the step size should be multiplied by a coefficient eta of $0 \sim 1$ and close to 1 to improve the accuracy of search.

(7) The above steps are repeated until an optimal solution is obtained.

The optimal location of longicorn beetles was output, and the corresponding scheduling scheme was obtained, thus obtaining the shortest scheduling time.

The algorithm flow chart is shown in Figure (1).

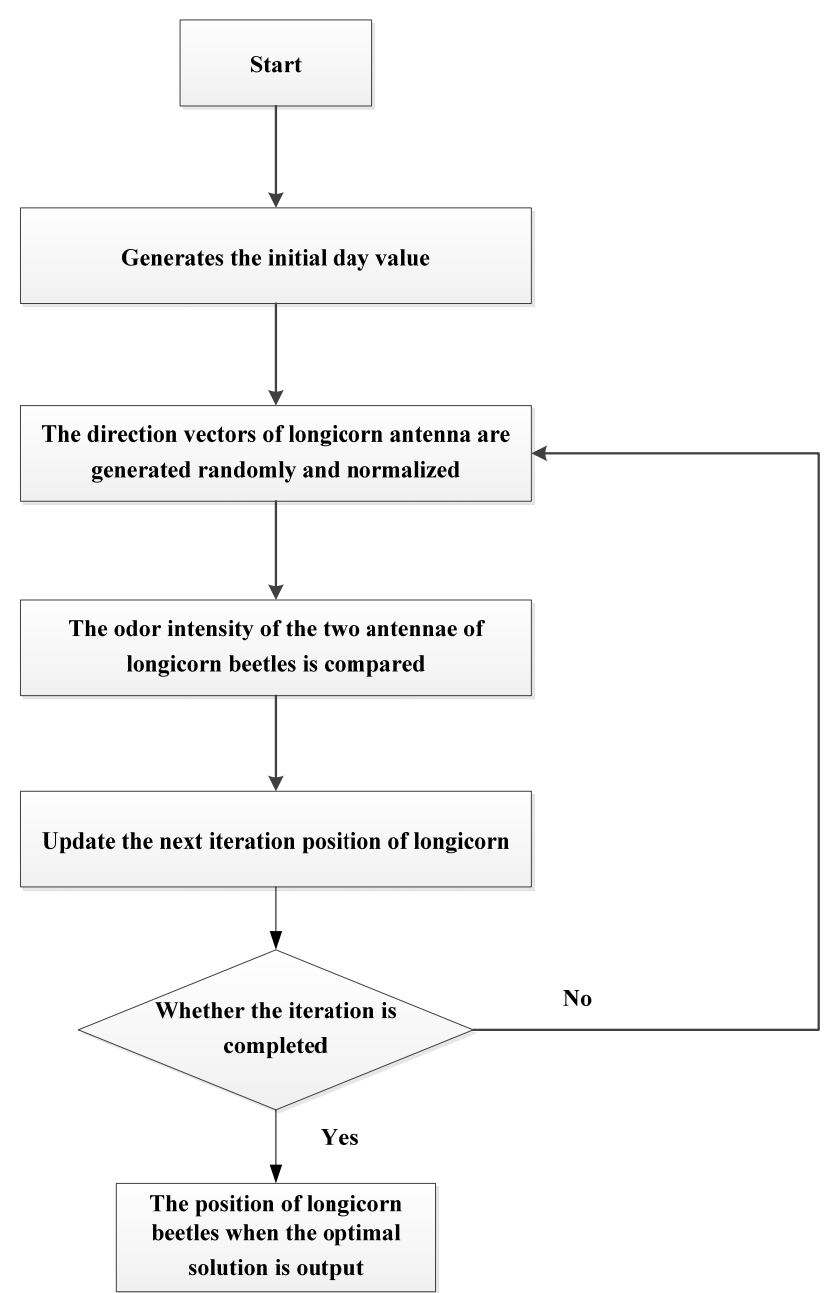

Fig 1. Algorithm Flow Chart

\section{Simulation experiment}

Due to the lack of actual cases consistent with the model in this paper, in order to verify the accuracy of the model, a simulation experiment is carried out in combination with relevant data in this paper. According to the statistics of the positions of dangerous situations in the northern sea area from 2003 to 2011, as shown in Figure (2)[10], two highoccurrence accident points are selected as simulation accident points, of which the coordinate of accident point 1 is $123.85^{\circ} \mathrm{E} 37.89^{\circ} \mathrm{N}$, and the coordinate of accident point 2 is $123.69^{\circ} \mathrm{E} 37.58^{\circ} \mathrm{N}$. According to the statistics of relevant literature, ships equipped in various ports are generally divided into two types: harbour tugs and harbour freight carrier. It is supposed that the ports participating in dispatching this time are Dalian Port, Yantai Port and Weihai Port, including 3 harbour tugs (serial number A1, A2, A3) and 2 harbour freight carriers (serial number B1, B2) in Dalian Port; 2 harbour tugs (serial number A4, A5) and 1 harbour freight carrier (serial number B3) in Yantai Port; 1 harbour tug (serial number A6) and 1 harbour freight carrier 
(serial number B4) in Weihai Port. Hubour tug volume is $300 \mathrm{t}$ and sailing speed is $8 \mathrm{n}$ mile; harbour transport cargo volume is $100 \mathrm{t}$ and speed is $20 \mathrm{n}$ mile.

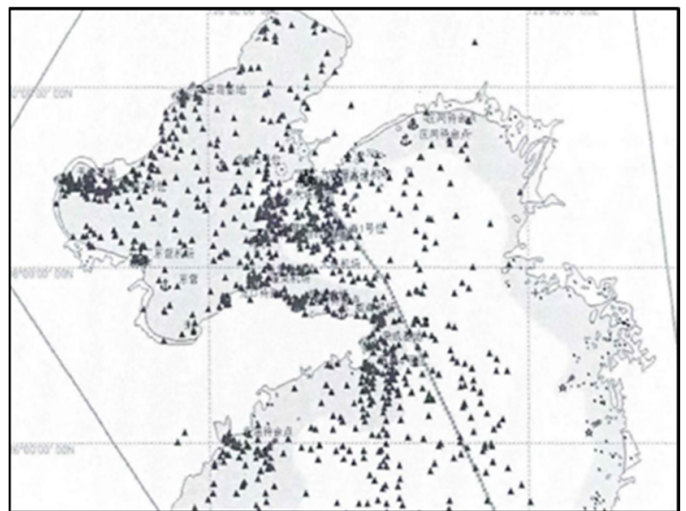

Fig 2. Location Map of Dangerous Accidents in Northern Seas from 2003 to 2011

The material demand range (ton) at the accident points is shown in Table (1).

Table1. Material Demand Range (ton) at the Accident Points

\begin{tabular}{|c|c|c|}
\hline Accident Point & Accident Point 1 & Accident Point 2 \\
\hline Material Range & {$[5100,7300]$} & {$[3500,6700]$} \\
\hline
\end{tabular}

The distance (nautical miles) from the supply point to the accident point is shown in Table (2).

Table2. Distance (nautical miles) from the Supply Point to the Accident Point

\begin{tabular}{|c|c|c|c|}
\hline Name & Dalian & Yantai & Weihai \\
\hline $\begin{array}{c}\text { Accident } \\
\text { Point 1 }\end{array}$ & 105 & 102 & 71 \\
\hline $\begin{array}{c}\text { Accident } \\
\text { Point 2 }\end{array}$ & 120 & 93 & 62 \\
\hline
\end{tabular}

Parameter setting: step size step $=300$, coefficient $c=2$, step size update coefficient $e t a=0.95$, maximum number of iterations $n=5000$. The algorithm is realized by programming in the environment of MTLAB 2018a. The algorithm is run for 1000 times and the optimal solution was obtained. The relationship between scheduling time and the number of iterations is shown in Figure (3).

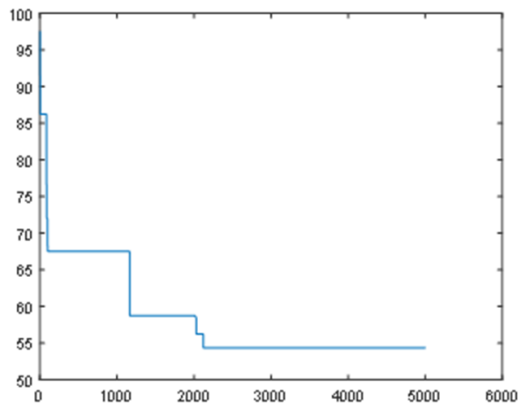

Fig 3. Algorithmic Iteration Graph
The iteration graph of the traditional BAS algorithm is shown in Figure (4).

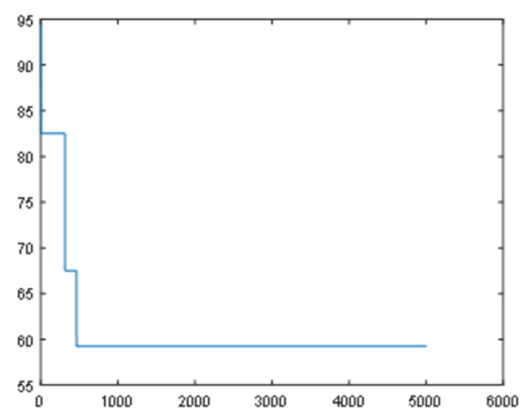

Fig 4. Algorithmic Iteration Graph

The optimal ship scheduling scheme is shown in Table (3).

Table3. Optimal Scheduling Scheme (tons)

\begin{tabular}{|c|c|c|}
\hline Number & Accident Point 1 & Accident Point 2 \\
\hline A1 & 225 & 565 \\
\hline A2 & 740 & 52 \\
\hline A3 & 740 & 61 \\
\hline A4 & 292 & 211 \\
\hline A5 & 566 & 174 \\
\hline A6 & 751 & 440 \\
\hline B1 & 827 & 32 \\
\hline B2 & 422 & 222 \\
\hline B3 & 372 & 651 \\
\hline B4 & 248 & 1190 \\
\hline
\end{tabular}

The shortest time is $54.375 \mathrm{~h}$. Since the one-way time is considered in the algorithm, the final result is $108.75 \mathrm{~h}$, and the running time of the algorithm is less than $0.2 \mathrm{~s}$. Compared with the two algorithms, although the traditional BAS algorithm converges faster than the variable step BAS algorithm, it is easy to fall into local optimum, and can not find the global optimal solution, but only to find the local optimal solution. Further analysis shows that it is precisely because of the introduction of the step update coefficient that the algorithm has a certain global search ability in the early stage, and reduces the step size when searching for a better solution, thereby improving the local search ability of the algorithm. Because the traditional BAS algorithm is easy to fall into local optimum, it requires repeated calculations more times to obtain a better solution, and compared with the BAS algorithm with variable step size, it is more timeconsuming, less efficient and the searched solution is worse, which is not conducive to rapid emergency response in complex maritime accidents. Therefore, the research on maritime emergency material dispatching based on variable step size BAS algorithm is of great significance to improve 
the emergency response capability, material dispatch efficiency and the success rate of maritime search and rescue.

\section{Conclusion and outlook}

In order to avoid unreasonable use of emergency resources and improve the efficiency of material dispatch, in this paper, considering to solve the problem of material dispatching after an emergency occurs at sea, aiming at minimizing the time of material dispatching and considering the different types of transport ships, a mathematical model of multiple accident points, multiple material supply points and multiple ships is established. In the process of using BAS algorithm, the step size update coefficient ETA is introduced to improve the global search ability and local search ability of the algorithm, and the optimization scheme of material dispatch is obtained, which improves the utilization efficiency of maritime emergency resources, saves scheduling time, and helps to improve the success rate of maritime search and rescue.

The simulation experiment mentioned in this paper is carried out under certain constraints, so some factors are not taken into account. For example, the accident point may drift under the influence of wind and waves, and the emergency response time of various ports may be different. All these factors will have an impact on the distribution of emergency supplies. Therefore, the influence of relevant factors should be considered in future related studies.

\section{References}

1. Fuyu Wang, Jie Ding. Research on the Optimization of Emergency Resource Scheduling Based on Improved Beetle Antennae Search Algorithm. Journal of Safety and Environment.J. Clerk Maxwell, A Treatise on Electricity and Magnetism, 3rd ed., vol. 2. Oxford: Clarendon, 1892, pp.68-73.
2. Jing Wang. Research on Dispatching Problem of Emergency Supplies in Marine Oil Spill Accident [D]. Dalian Maritime University, 2011.

3. Bo Shen. Research on Improvement and Application of Beetle Antennae Search Algorithm [D]. Shanghai: Donghua University, 2020.

4. Jun Wang. Research on Optimal Allocation of Water Rescue Resources [D]. Dalian: Dalian Maritime University, 2011.

5. Tingting Chen, He Yin, Hongli Jiang, Lu Wang. Portfolio Problem Solved by Particle Swarm Optimization Algorithm Based on Beetle Antennae Search. Computer system applications, 2019,28(2):171-176. http://www.c-s-a.org.cn/10033254/6771.html

6. Meirong Wang. Research on Dispatching of Emergency Resources for Maritime Accidents and Disasters[D]. Dalian: Dalian Maritime University, 2010

7. Hui Zhao, Mengya Hao, Hongjun Wang, Youjun Yue. Path Planning Method for Agricultural Robot Based on Improved A* Algorithm and Beetle Antennae Search Algorithm [J].Science Technology and Engineering,2019,19(31):185-190

8. Xue Binzhu, Jing Lu. A Two-stage Cooperative Dispatching Model for Marine Emergency Supplies [J]. Shipping Management, 2020,42 (05) : 38-42

9. QingChen Sun, LinChi Qu.Location Model Considering the Uncertain Demand of Multiple Emergency Supplies [J].Journal of Guangxi University (Natural Science Edition), 2019,44 (02): 448-454

10. Haipeng Du. Research on the Allocation of Professional Maritime Rescue Forces of Countries in the Northern Waters[D].Dalian: Dalian Maritime University, 2012 